\title{
Properties of the propagating shock wave in the accretion flow around GX 339-4 in the 2010 outburst (Research Note)
}

\author{
D. Debnath ${ }^{1}$, S. K. Chakrabarti ${ }^{1,2}$, and A. Nandi ${ }^{1,3}$ \\ 1 Indian Centre for Space Physics, Chalantika 43, Garia Station Rd., Kolkata 700084, India \\ 2 S. N. Bose National Centre for Basic Sciences, Salt Lake, Kolkata 700098, India \\ 3 On deputation from Indian Space Research Organization-HQ, Bangalore, India \\ e-mail: [dipak;anuj]@csp.res.in, chakraba@bose.res.in
}

Received 14 May 2010 / Accepted 7 September 2010

\begin{abstract}
Context. The black hole candidate GX 339-4 exhibited an X-ray outburst in January 2010, which is still continuing. We here discuss the timing and the spectral properties of the outburst using RXTE data.

Aims. Our goal is to study the timing and spectral properties of GX 339-4 using its recent outburst data and extract information about the nature of the accretion flow.

Methods. We use RXTE archival data of the recent GX 339-4 outburst and analyze them with the NASA HEAsoft package, version 6.8. We then compare the observed quasi-periodic oscillation (QPO) frequencies with those from existing shock oscillation model and obtain the nature of evolution of the shock locations during the outburst.

Results. We found that the QPO frequencies are monotonically increasing from $0.102 \mathrm{~Hz}$ to $5.69 \mathrm{~Hz}$ within a period of $\sim 26$ days. We explain this evolution with the propagating oscillatory shock (POS) solution and find the variation of the initial and final shock locations and strengths. The model fits also give the velocity of the propagating shock wave, which is responsible for the generation of QPOs and their evolutions, at $\sim 10 \mathrm{~m} \mathrm{~s}^{-1}$. We observe from the spectra that up to 2010 April 10, the object was in a hard state. After that, it went to the hard-intermediate state. On April 18, it had a state transition and went to the soft-intermediate state. On May 15, another state transition was observed and the source moved to the soft state.

Conclusions. As in the previously fitted outburst sources, this source also showed the tendency of a rapidly increasing QPO frequency $\left(v_{\mathrm{QPO}}\right)$ on a viscous time scale, which can be modeled quite accurately. In this case, the shock seems to have disappeared at about $\sim 172$ Schwarzschild radii, unlike in the 2005 outburst of GRO J1655-40, where the shock disappeared behind the horizon.
\end{abstract}

Key words. accretion, accretion disks - black hole physics - Shock waves - stars: individual: GX 339-4 - X-rays: binaries

\section{Introduction}

The source GX 339-4 is a well known stellar-mass Galactic black hole candidate. This bright variable X-ray source was first observed during the survey period from October 1971 to January 1973 by the MIT X-ray detector on-board the OSO-7 satellite in the energy range of 1-60 keV. GX 339-4, a transient low-mass X-ray binary (LMXB) system located at $(l, b)=$ $\left(338^{\circ} .93,-4.27\right)$ (Markert et al. 1973) with RA $=17^{\mathrm{h}} 02^{\mathrm{m}} 49^{\mathrm{s}} .36$ and Dec $=-48^{\circ} 47^{\prime} 22^{\prime \prime} 8$ (J2000). The optical spectroscopic study indicates that the mass function of the source is $M=$ $5.8 \pm 0.5 M_{\odot}$ and the distance $D=6 \mathrm{kpc}$ (Hynes et al. 2003, 2004).

Since its discovery, GX 339-4 has undergone several outburst phases, during which the source was observed in different wavebands to reveal the nature in multiple wavelengths (Liu et al. 2001; Homan et al. 2005). During the RXTE era (1996 onward), this source exhibited frequent X-ray outbursts $(1998,2002 / 2003,2004 / 2005,2006 / 2007)$ at a 2-3 years of interval with very low luminosity states in between each episode. The complex outburst profile in each epoch generally begins and ends in the low/hard state, which is quite common in other outburst candidate of black holes (e.g., GRO J1655-40, XTE J1550-564). This general behavior is understood to be caused by sudden variation of viscosity in the system (Mandal \& Chakrabarti 2010), which in turn causes the accretion rate of the standard Shakura-Sunyaev (1973) disk (hereafter referred to as the Keplerian rate) to rise and possibly makes the inner edge move in. These transient black hole candidates show low and intermediate frequency quasi-periodic oscillations (QPOs) in their power density spectra. In general, during the rising hard state of the outburst the frequency of the QPO increases, whereas during the declining phase, the QPO frequency is gradually decreased. The QPO evolution in these objects can be well understood through the propagating oscillatory shocks (POS; Chakrabarti et al. 2005, 2008, 2009). Though several studies of the evolution of the temporal and spectral states of GX 339-4 during the previous outbursts were carried out (Nowak et al. 1999; Belloni et al. 2005; Motta et al. 2009), the underlying physical processes remained unclear. Our attempt here is to see if the POS solution of our group can also explain the present outburst. Note that for the traditional soft-X-ray transients with fast rise and exponential decay (FRED) lightcurve with typically long recurrence times, there are so-called disk instability models (Cannizzo 1993; Lasota 1996) where matter also moves in owing to viscous processes. However these models do not address variations of QPOs. 
Recently, after remaining in the quiescent state for three long years (except for a short spell of very weak activity in 2009 as observed in SWIFT/BAT), GX 339-4 became X-ray-active again on 2010 January 03, with a first detection by MAXI/GSC onboard HETE (Yamaoka et al. 2010). Immediately after the announcement of the X-ray trigger, RXTE started monitoring the source from 2010 January 12 (Tomsick 2010). During the initial outburst phase, the source was in the low-hard state without any signature of QPO in the power density spectrum (PDS). In this outburst phase we first observed the QPO at $102 \mathrm{mHz}$ on 2010 March 22 (MJD 55 277). After that, the QPO frequency monotonically increased to $5.69 \mathrm{~Hz}$ until 2010 April 17 (MJD 55 303). Afterward, QPOs were sporadically on and off (e.g., $5.739 \mathrm{~Hz}$, $5.677 \mathrm{~Hz}$ and $5.913 \mathrm{~Hz}$ on April 18, 22 and 29 respectively), always remaining at about the same value. These sporadically appearing QPOs in PDS were observed until 2010 May 14. The observed QPOs in hard and hard-intermediate states (Homan \& Belloni 2005) are of the " $C$ " type and in soft-intermediate state are of the "B" type (van der Klis 2004; Casella et al. 2005).

The gradual increase of the QPO frequency in the rising phases of transient black hole and neutron star candidates have been known for a long time (e.g., Belloni \& Hasinger 1990; Belloni et al. 2002; Maitra \& Bailyn 2004). In the present context of studying outbursting black hole candidates, evolutions of QPOs during the 2005 outburst of GRO J1655-40 (Chakrabarti et al. 2008, hereafter CDNP08) and the 1998 outburst of XTE J1550-564 (Chakrabarti et al. 2009, hereafter CDP09) showed the monotonically increasing behavior in the rising phase like the one we find here in GX 339-4. However, while in GRO J1655-40 the QPO disappeared completely and then re-appeared after about six months (Debnath et al. 2008) and in XTE J1550-564, $v_{\mathrm{QPO}}$ started declining immediately after reaching maximum (CDP09), in the present case, $v_{\mathrm{QPO}}$ began to stall at about 5.7-5.9 Hz. In CDNP08 and CDP09, it was shown that the oscillating shock that produces the QPOs was required to move in at a roughly constant speed of about $20 \mathrm{~m} \mathrm{~s}^{-1}$. In both cases, a clear picture emerged about the system: it was found that while the low-angular momentum transonic flow (hereafter referred to as the sub-Keplerian matter, see, e.g. Chakrabarti 1990) was always present even in the quiescent state, the Keplerian disk moved in closer to the black hole in the rising phase and then receded far away in the declining phase. This picture was corroborated when even the hardness-intensity diagram was reproduced (Mandal \& Chakrabarti 2010) with this consideration.

We here examine the nature of the rising phase of GX 3394 and show how it changed from hard state to soft state via a short-lived hard-intermediate and soft-intermediate state. We also show that the evolution of QPOs can be understood by the POS solution as in the case of other outbursts. In the next section, we present the observational results of GX 339-4 since it showed evidences of the outburst in January, 2010. We also fit the QPOs using POS solution presented in CDNP08 and CDP09. Finally, in Sect. 3, we present the concluding remarks.

\section{Observational results and analysis}

We now present the timing and spectral properties of the GX 339-4 X-ray outburst using the archival data of the RXTE PCA and ASM instruments. We use the standard RXTE data analysis software package HEAsoft 6.8. For RXTE/ASM (Levine et al. 1998), one day averaged archival data of the different energy bands (2-3, 3-5, 5-12 \& 2-12 keV) were downloaded and analyzed. For the PCA data (Jahoda et al. 1996) analysis, we mainly use the most stable and well conditioned

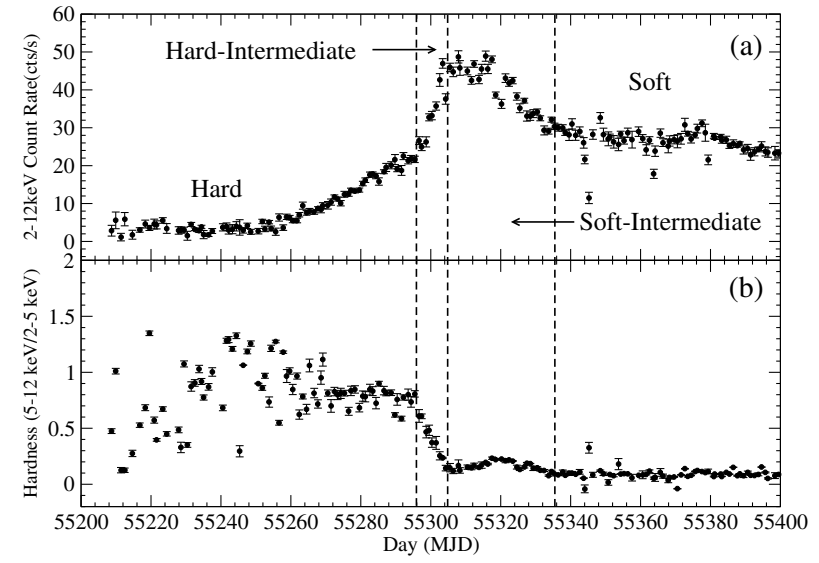

Fig. 1. a) 2-12 keV ASM lightcurve and b) hardness ratio (5-12 keV vs. $2-5 \mathrm{keV}$ count ratio) as a function of the MJD of the observation. The vertical dashed lines at MJD 55 296, MJD 55 304, and MJD 55331 indicate the state transitions from hard to hard-intermediate state, then to soft-intermediate state and finally to soft state.

proportional counter unit 2 (PCU2) data (all six layers). Background spectra were made using FTOOLS runpcabackest task and the most recent bright source model. The task pcarsp was used to generate the PCA response file. For the timing analysis, we used the PCA Event mode data with a maximum timing resolution of $125 \mu \mathrm{s}$, and for the spectral analysis we used the PCA "standard 2" data. In the entire PCA data analysis, we did not include the deadtime corrections because the counts are not very high, the maximum rate being around $1200 \mathrm{cts} / \mathrm{s}$. We verified that the error caused by this is at most $4 \%$. In Fig. 1a,b, we present the ASM lightcurve and the hardness ratio as a function of days. Sudden changes in slope on MJD 55 296, MJD 55304 , and MJD 55331 are indications of the state transitions. From the hardness ratio (Fig. 1b) we see that the spectrum was hard till MJD 55 296. At MJD 55304 the spectrum became softer very quickly. This is the so-called hard-intermediate state (Homan $\&$ Belloni 2005). After that, the ratio remained almost constant. Sporadic QPOs (see below) in this state indicate that the object is in the soft-intermediate state. Around 2010 May 15 (MJD 55331), the source moved to the soft state, with a sharp fall in the count rate in the $4-15 \mathrm{keV}$ energy range. In Fig. 2 we show the total counts $(2-20 \mathrm{keV})$ as a function of the hardness ratio $H R=(6-20 \mathrm{keV}) /(2-6 \mathrm{keV})$ from 2010 January 12 (MJD 55 208) until August 14 (MJD 55 422). Four phases are clear: in the range $\mathrm{A}$ to $\mathrm{B}$, the object is in the hard state, in the range $B$ to $C$, the object is in the hard-intermediate state, $C$ to $D$, the object is in the soft-intermediate state and beyond D, the object is in the soft state. A detailed physical picture will be discussed below in Sect. 2.2.

\subsection{Timing analysis}

We analyze 114 observational IDs from 2010 January 12 $(\mathrm{MJD}=55208)$ to 2010 July 8 (MJD = 55 385). For the timing analysis, we use RXTE ASM and PCA public data. In the study of the temporal properties of any black hole candidate, finding quasi-periodic oscillations (QPOs) in power density spectra (PDS) is as important as the observation of the photon count/flux variations. To generate the PDS, we used the "powspec" task of XRONOS package with a normalization factor of " -2 " to have the "white" noise subtracted rms fractional variability on 


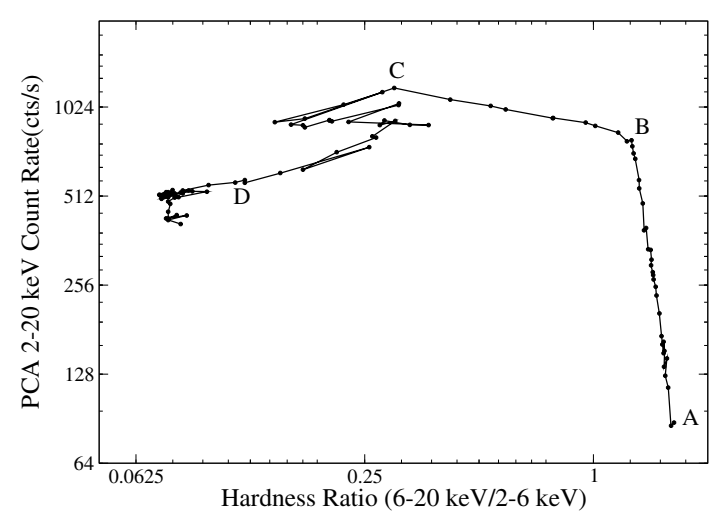

Fig. 2. Hardness intensity diagram (HID) of GX 339-4 observed with RXTE/PCA from 2010 January 12 to August 14, as it approaches the soft state from the hard state via hard-intermediate and softintermediate spectral states. The total count rates in the $2-20 \mathrm{keV}$ energy band along $Y$-axis and the ratio of the count rates in the $6-20 \mathrm{keV}$ and $2-6 \mathrm{keV}$ bands are given on the $X$-axis. The points A, B, C, and D are on MJD 55 208, MJD 55 296, MJD 55 304, and MJD 55331 respectively. Point A indicates our first observation day and points B, C, and D indicate the state transitions.
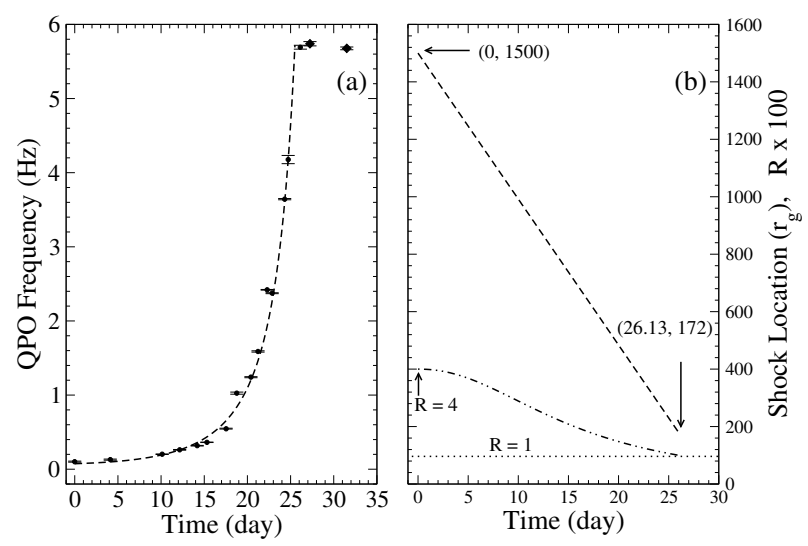

Fig. 3. a) Variations of the QPO frequency with time (in day) of the rising phase of the outburst with the fitted POS model (dotted curve) are shown. The diamonds indicate the last two incidents of observed QPOs (on 2010 April 18 and 22) and they are not included in our model fitting, because the shock already achieved its weakest strength of $R \sim 1$ earlier on April 17. b) Variation of the shock location (in $r_{\mathrm{g}}$ ) and shock strength $(R)$. The shock seems to be stagnating at around 172 Schwarzschild radii.

2-15 keV (0-35 channels) PCU2 lightcurves of 0.01 s time bins. The power obtained has the unit of $\mathrm{rms}^{2} / \mathrm{Hz}$. Quasiperiodic oscillations are generally of a Lorentzian type (Nowak 2000; van der Klis 2005) and thus these are fitted with model Lorentzians. In Fig. 3a we show the variation of the QPO frequency in this period. The monotonically increasing frequency (starting from $102 \mathrm{mHz}$ observed on March 22 to $5.69 \mathrm{~Hz}$ observed on April 17) as in GRO J1655-40 and XTE J1550-564 (CDNP08, CDP09) motivated us to fit it with the same POS solution as used in CDNP08 and CDP09. In this solution, at the onset of the outburst, a shock wave moves toward the black hole, which oscillates either because of resonance (cooling time $\sim$ infall time; Molteni et al. 1996) or because of the fact that the Rankine-Hugoniot relation is not satisfied (Ryu et al. 1997) to form a steady shock. The QPO frequency is obtained from the inverse of the in-fall time scale from the post-shock region. The oscillation of the shock produces the oscillation of hard X-ray intensity, because the post-shock flow behaves like a Compton cloud that intercepts a variable number of soft photons during oscillations. The governing equations are in CDNP08 and will not be repeated here. By fitting the QPO frequencies with POS solution we find that the compression ratio $R=\rho_{-} / \rho_{+}$monotonically goes down from $R_{0}=4$ (strongest shock) to $\sim 1$ (weakest shock) through the relation $1 / R \rightarrow 1 / R_{0}+\alpha\left(t_{\mathrm{d}}\right)^{2.15}$, where, $t_{\mathrm{d}}$ is the time in days (taking the first day of the QPO observation as the 0th day, i.e., from March 22). Here, $\alpha$ is a constant that determines how rapidly the shock strength decreases with time. The value of $\alpha$ is obtained by the constraint that on the last day (i.e., after $t_{r} \sim 26$ on April 17) the QPO was observed, the shock became the weakest $(R \sim 1)$. Using this condition that $R \rightarrow 1$ on $t_{\mathrm{d}}=t_{r}$, we obtain $\alpha$ at $6.8 \times 10^{-4}$ in the present case. In Fig. 3b we show the fitted shock strength and the shock location as a function of day, where 0 is March 22 when the QPO was first detected. According to our fit, the shock started at $r \sim 1500$ Schwarzschild radii $\left(r_{\mathrm{g}}=2 G M / c^{2}\right)$ and disappeared on the 26th day (April 17), when it was at 172 Schwarzschild radii. The shock wave is found to move toward the black hole at a constant velocity of $\sim 10 \mathrm{~m} \mathrm{~s}^{-1}$, somewhat slower that the other two members, namely, GRO J1655-40 and XTE J1550-564 where the velocity was about twice as high. After that, the QPO is seen sporadically, but the frequency remains about the same. This is because when the shock is weakest, moving inward cannot reduce its strength any more. During this period, the Keplerian matter also moves in, increasing the softness of the spectrum (Fig. 1). In the soft-intermediate state, the Keplerian rate rises to become comparable to the sub-Keplerian rate, while in the soft state (i.e., current spectral state) the Keplerian rate dominates. The net duration of the soft-intermediate and soft states would therefore depend on how long the viscosity remains sufficient high to maintain a Keplerian flow. Thus the prediction of the net duration of the outburst is not easy. Detailed modeling is required to predict the possible duration of any outburst.

\subsection{Spectral analysis}

For the spectral study we use 3-25 keV PCA "standard-2" data of PCU2 and XSPEC (version 12.5) package. For all observations we kept the hydrogen column density $\left(N_{\mathrm{H}}\right)$ fixed at $5 \times 10^{21} \mathrm{~cm}^{-2}$ (Mota et al. 2009), using the absorption model wabs. All the spectra were fitted with standard disk blackbody and power-law models. For each spectrum one Gaussian line at $\sim 6.5 \mathrm{keV}$ was used. For the best fit, we also added $1.0 \%$ systematic error to the full spectrum. In Fig. $4 a-d$ we show the evolution of photon counts and the photon index. We show the results from March 5 onward. Panel (a) shows very hard photons in the 15-30 keV range, which could come only from Comptonization; (b) shows the photon counts of the intermediate energy range 4-15 keV, which is monotonically increasing, and the panel (c) shows very soft photons $(2-4 \mathrm{keV})$, which could come only from the blackbody component, i.e., the Keplerian disk. Panel (d) shows the photon index $\Gamma$. Note that QPOs are observed immediately after a "kink" occurring on MJD 55 274, the physics which is still unclear. $\Gamma$ remains less than 2 , and the very hard photons increase until MJD 55 296. We may assume that the object is in the hard state. After MJD 55296 , the hard photon count rapidly diminishes and the soft photon count rapidly increases. In this phase, the Keplerian rate increases and becomes comparable to the sub-Keplerian rate. As Chakrabarti \& Titarchuk (1995) pointed out, the spectral index becomes very sensitive 


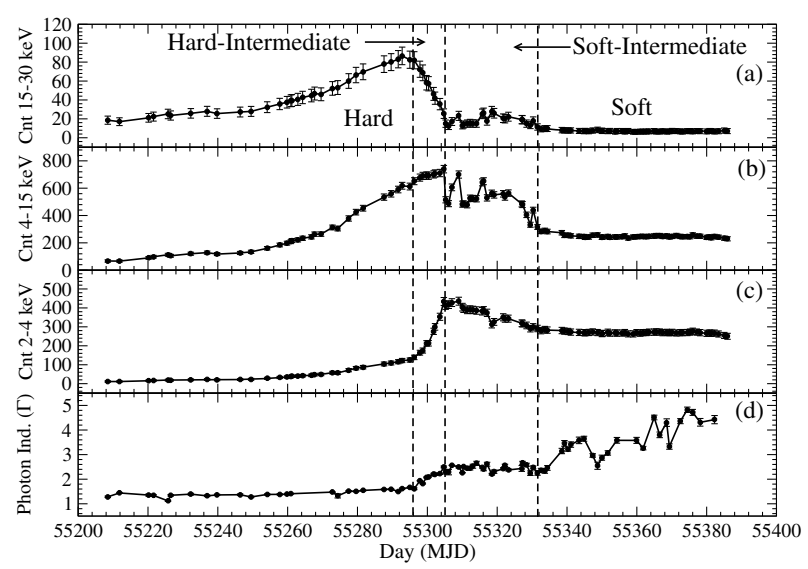

Fig. 4. Variation of the RXTE/PCA count rates in a) $15-30 \mathrm{keV}$; b) $4-15 \mathrm{keV}$; and c) $2-4 \mathrm{keV}$ energy bands; and d) the power-law photon index $\Gamma$ with day (in MJD) are plotted. The vertical dashed lines are on MJD 55 296, MJD 55304.7 and MJD 55 331, indicating the state transitions from hard to hard-intermediate, to soft-intermediate and to soft states respectively.

to the Keplerian rate when the rates are comparable at around $\sim 0.5-1$ Eddington rate. This is the intermediate state. The index rapidly increased to about 2.5 on MJD 55304.7 and then remained almost constant, perhaps because the Keplerian disk rate had reached its peak and was sustained by the viscosity. In future, when the viscosity is reduced, the Keplerian rate would decrease and the object will gradually go back to the hard state. With a very low Keplerian rate, the inner part of the disk evaporates, which is equivalent to saying that its inner edge has receded.

\section{Discussions and concluding remarks}

We analyzed the recent outburst of the black hole candidate GX 339-4. We studied the evolution of the spectral and timing properties since 2010 January 12 till 2010 July 8 and showed that there was no signature of QPO till March 21. After this, until April 17, QPO was continuously observed. The frequency was monotonically increasing in a very similar way to what was shown in GRO J1655-40 and XTE J1550-564. Until April 9, the spectral photon index was less than 1.6, the source was at a pure hard state. Then the object moved to the hard-intermediate state as the Keplerian rate started increasing when the photon spectral index changed from $\sim 1.6$ to $\sim 2.0$. This short duration state was continued until April 17. Then as the Keplerian rate became comparable to the sub-Keplerian rate, the spectral index rapidly became soft $(\Gamma \sim 2.5)$ and the object remained in the soft-intermediate state for $\sim 26$ days until 2010 May 14. Here sporadic QPOs were observed and the QPO frequency remained at around $6 \mathrm{~Hz}$. After that the Keplerian rate dominates and the spectrum becomes softer with a high spectral index $(\Gamma>3.0)$. We believe that when the viscosity is reduced in future, the Keplerian rate will be so much reduced that the Keplerian disk may itself evaporate and the inner edge of the Keplerian could be thought to have receded from the black hole. In this case, the object would return to the hard state (Mandal \& Chakrabarti 2010) and monotonically decreasing nature of the QPO frequencies will be observed as predicted by the POS solution (CDNP08, CDP09).
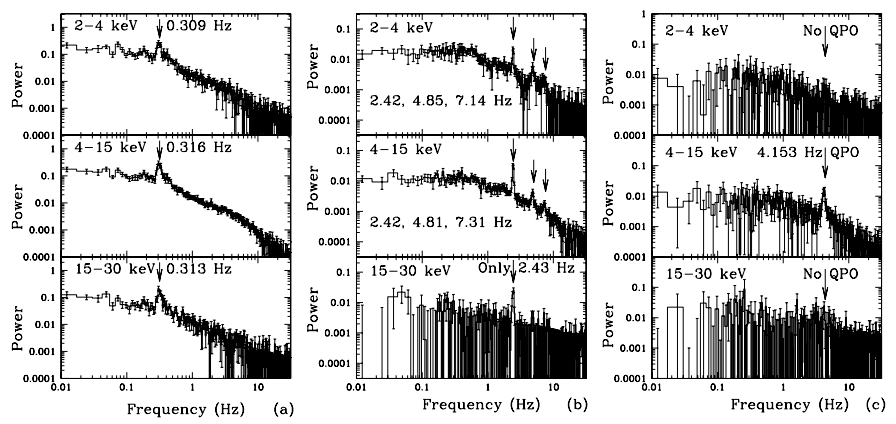

Fig. 5. a)-c): Power density spectrum of photons in three different energy bands in three different observational IDs: a) Obs. ID:95409-0113-02 of 2010 April 5, b) Obs. ID:95409-01-14-06 of 2010 April 13; and c) Obs ID:95409-01-15-00 of 2010 April 16. The top, middle, and bottom panels are for 2-4 keV, 4-15 keV, and 15-30 keV bands respectively. Shock locations are found to be at $780 r_{\mathrm{g}}, 367 r_{\mathrm{g}}$, and $244 r_{\mathrm{g}}$, and the compression ratios (which represent the shock strength) are $2.20,1.27$, and 1.08 respectively. In a) $2-4 \mathrm{keV}$ photons are actually Comptonized photons, they also show strong QPOs, while in c) they are soft photons and do not show QPOs (Chakrabarti \& Manickam 2000). Note also that because the shock is stronger in a) the rms amplitude is higher in all three energy bands, while in c) the shock is weaker and the rms is also very low. In b), the fundamental and two harmonics could be seen.

In Chakrabarti \& Manickam (2000), the authors showed that the true "soft photons" i.e., the photons emitted from the preshock flow, do not exhibit QPOs. A cross-check that the twocomponent model is valid and that the QPO is caused by the oscillation of the Comptonized cloud (namely, the post-shock region) can be made. As the shock moved in, the post-shock flow was heated up roughly as $T \sim 1 / r$ while the pre-shock Keplerian flow was heated up as $r^{-3 / 4}$ (Shakura \& Sunyaev 1973). This means that so-called "soft-photons" in $2-4 \mathrm{keV}$ range could be actually Comptonized photons at a large distance and should be treated as hard photons. Thus, $2-4 \mathrm{keV}$ photons should show QPOs at a large distance only. In Fig. 5a-c we plot the power density spectra of photons in the $2-4 \mathrm{keV}, 4-15 \mathrm{keV}$ and 15-30 keV bands on the April 5, 13 and 16 respectively when the shock (Compton cloud boundary) was located at $\sim 780 r_{\mathrm{g}}, 367 r_{\mathrm{g}}$ and $244 r_{\mathrm{g}}$ respectively with compression ratios of 2.2, 1.27 and 1.08 respectively. The spectral and timing properties of the object in and around these days are presented in Tables 1 and 2. In Table 1 we show how the photon index and fluxes vary in these days. $\chi^{2}$ and the number of degrees of freedom (d.o.f.) in the spectral fits are also included. In Table 2 we note that the rms amplitude of the fundamental QPO decreased with time in 2-4 keV range. This shows that the number of Comptonized photon is decreasing in this energy bin and increasing in the $4-15 \mathrm{keV}$ bin as the shock moves in. We note that in the $4-15 \mathrm{keV}$ range, the rms initially decreased and then increased. The latter increase is because the entire Comptonized photons now belong to this energy bin. In high-energy channels (15-30 keV) the rms amplitude decreased because of the paucity of photons.

Clearly Table 2 shows that the rms amplitude was highest in all energy ranges when the shock was farther out. This shows that the oscillation of the Compton cloud, which was caused by a stronger shock at a large distance, was high enough to significantly modulate the outgoing flux. This is particularly surprising in the 2-4 keV range, because it was supposed to be the energy of the "soft-photons". This shows that far out, these photons are actually Comptonized photons. Note that the QPO 
Table 1. Spectral properties during the initial outburst phase.

\begin{tabular}{|c|c|c|c|c|c|}
\hline \multirow[t]{2}{*}{ Obs. Id" } & \multirow{2}{*}{$\begin{array}{l}\text { UT } \\
\text { Date }\end{array}$} & \multirow{2}{*}{$\begin{array}{c}\text { Photon } \\
\text { Index }(\Gamma)\end{array}$} & \multicolumn{2}{|c|}{ Flux $^{\dagger}$} & \multirow[t]{2}{*}{$\chi^{2} /$ d.o.f.* } \\
\hline & & & $3-10 \mathrm{keV}$ & $10-25 \mathrm{keV}$ & \\
\hline $\mathrm{X}-10-05$ & $2010-03-17$ & 1.473 & 2.533 & 2.868 & $39.92 / 48$ \\
\hline X-13-02 & $2010-04-05$ & 1.504 & 2.405 & 2.545 & $55.13 / 48$ \\
\hline$X-14-06$ & $2010-04-13$ & 2.073 & 6.495 & 3.637 & $43.84 / 48$ \\
\hline$X-15-00$ & $2010-04-16$ & 2.185 & 3.446 & 1.390 & $68.51 / 48$ \\
\hline X-16-04 & $2010-04-28$ & 2.613 & 6.238 & 0.658 & $56.74 / 48$ \\
\hline
\end{tabular}

Notes. ${ }^{(\dagger)}$ Flux in unit of $10^{-9} \mathrm{erg} \mathrm{cm}^{-2} \mathrm{~s}^{-1}$ ( $^{(*)}$ Here, $X=95409-01$. ${ }^{(* *)}$ d.o.f. means no. of degrees of freedom.

Table 2. Timing properties during the initial outburst phase.

\begin{tabular}{ccccc}
\hline \hline \multirow{2}{*}{ Obs. Id } & \multirow{2}{*}{$\mathrm{UT}$} & \multicolumn{3}{c}{$v_{\mathrm{QPO}}$ (Hz) and rms amp. $(\%)$} \\
\cline { 3 - 5 } & Date & $2-4 \mathrm{keV}$ & $4-15 \mathrm{keV}$ & $15-30 \mathrm{keV}$ \\
\hline $\mathrm{X}-10-05$ & $2010-03-17$ & - & - & - \\
$\mathrm{X}-13-02$ & $2010-04-05$ & $0.309,15.190$ & $0.316,16.048$ & $0.313,11.893$ \\
$\mathrm{X}-14-06$ & $2010-04-13$ & $2.420,7.095$ & $2.424,8.258$ & $2.430,7.292$ \\
& & $4.846,6.056$ & $4.813,5.585$ & - \\
& & $7.139,4.241$ & $7.310,4.987$ & - \\
X-15-00 & $2010-04-16$ & - & $4.153,10.293$ & - \\
X-16-04 & $2010-04-28$ & - & - & - \\
\hline
\end{tabular}

Notes. ${ }^{(*)}$ Here, $X=95409-01$.

is absent in this range on April 16. This is expected, because the Compton cloud is so close to the black hole that these photons are contributed mostly from the Keplerian flow and are not modulated. Note that the appearance of harmonics (Yu 2010) depends on the energy of the photons. On the April 16, the shock is very weak and a faint QPO is seen only in $4-15 \mathrm{keV}$. At lower energy bins QPO was not seen because they were emitted from the pre-shock flow, and at higher energy bins, the number of photons was statistically insignificant. This analysis indicates that the general two-component solution of the outburst sources as developed in CDNP08, CDP09, and Mandal \& Chakrabarti (2010) can explain most of what is seen in GX 339-4 so far. Assuming that this outburst is similar to that in GRO J1655-40 (CDNP08) we expect that once the object goes to the low-hard state, it will take about 32-38 days to return to quiescence and the QPO frequency would monotonically decrease from $\sim 6 \mathrm{~Hz}$ to a few $\mathrm{mHz}$ in that period.

Miller et al. (2006), Ramadevi \& Seetha (2007), and Rykoff et al. (2007) recently argued that the presence of a thermal emission even in the low-hard state of some outburst sources points to a Keplerian disk with an inner edge very close to the black hole. The objects exhibit a behavior similar to a classical outburst with FRED-type lightcurve. In these cases, the data in the rising phase are scarce and these authors used the data from the day when the outburst was near its peak and had a softer spectrum. The conclusions of these authors do not necessarily mean that the Keplerian disk cannot move in within the viscous time scale as in our paradigm. According to our picture, the Keplerian disk has already moved in during the rising part and was present when these authors commenced their analysis.

Black hole accretion is a complex process, and it is abundantly clear that a simple standard disk (Shakura \& Sunyaev 1973 ) is not capable of explaining most of the observations. Out of all the observations, the outbursting sources are extremely important, because they exhibit the changes in spectral states in rapid succession. Similarly they also generally exhibit a systematic variation of QPO frequencies. These enable us to study the dynamics of matter close to a black hole. A number of outbursting sources, whether they exhibit FRED lightcurves as in the soft X-ray transients or slow-rise and slow-decay (SRSD) lightcurves as in GX 339-4 or GRO J1655-40 show similar and timing properties. Our two-component flow paradigm seems to be capable of explaining these sources quite naturally if one assumed that the outburst is caused by a rapid rise of viscosity, which drives Keplerian flows towards the black hole and/or converts some of the low angular momentum flows into Keplerian flows. Because quantifying viscosity is not easy, a prediction of a detailed behavior has not been possible so far. However, analyses of these sources are very useful in advance our quest for a general solution of this difficult problem.

Acknowledgements. D. Debnath acknowledges the support of CSIR-NET scholarship.

\section{References}

Belloni, T., \& Hasinger, G. 1990, A\&A, 230, 103

Belloni, T., Colombo, A. P., Homan, J., Campana, S., \& van der Klis, M. 2002, A\&A, 390, 199

Belloni, T., Homan, J., Casella, P., et al. 2005, A\&A, 440, 207

Cannizzo, J. 1993, in Accretion Discs in Compact Stellar Systems, ed. J. C. Wheeler (Singapore: World Scientific), 6

Chakrabarti, S. K. 1990, Theory of Transonic Astrophysical Flows (Singapore: World Scientific)

Chakrabarti, S. K., \& Titarchuk, L. G. 1995, ApJ, 455, 623

Chakrabarti, S. K., \& Manickam, S. G. 2000, ApJ, 531, L41

Chakrabarti, S. K., Nandi, A., Debnath, D., Sarkar, R., \& Datta, B. G. 2005, IJP 79(8), 841 [arXiv: astro-ph/0508024]

Chakrabarti, S. K., Debnath, D., \& Nandi, A., \& Pal, P. S. 2008, A\&A, 489, L41 (CDNP08)

Chakrabarti, S. K., Dutta, B. G., \& Pal, P. S. 2009, MNRAS, 394, 1463C (CDP09)

Casella, P., Belloni, T., Homan, J., \& Stella, L. 2005, A\&A, 426, 587

Debnath, D., Chakrabarti, S. K., Nandi, A., \& Mandal, S. 2008, BASI, 36, 151

Hynes, R. I., Steeghs, D., Casares, J., Charles, P. A., \& O’Brien, K. 2003, ApJ, 583, L95

Hynes, R. I., Steeghs, D., Casares, J., Charles, P. A., \& O’Brien, K. 2004, ApJ, 609,317

Homan, J., \& Belloni, T. 2005, Ap\&SS, 300, 107

Homan, J., Buxton, M., Markoff, S., et al. 2005, ApJ, 624, 295H

Lasota, J.-P. 1996, in Compact Stars in Binaries, ed. van Paradijs, J., van den Heuvel, E. P. J., Kuulkers, E., Proc. IAU Colloq., 165, 43 (Dordrecht: Kluwer) Liu, Q. Z., van Paradijs, J., \& van den Heuvel, E. P. J. 2001, A\&A, 368, 1021

Maitra, D., \& Bailyn, D. 2004, ApJ, 608, 444

Mandal, S., \& Chakrabarti, S. K. 2010, ApJ, 710, L147

Markert, T. H., Canizares, C. R., Clark, G. W., et al. 1973, ApJ, 184, L67

Miller, J. M., Homan, J., \& Miniutti, G. 2006, ApJ, 652, L113

Molteni, D., Sponholz, H., \& Chakrabarti, S. K. 1996, ApJ, 457, 805

Motta, S., Belloni T., \& Homan J. 2009, MNRAS, 400, 1603

Nowak, M. A. 2000, MNRAS, 318, 361

Nowak, M. A., Wilms, J., \& Dove, J. B. 1999, ApJ, 517, 355

Ramadevi, M. C., \& Seetha, S. 2007, MNRAS, 378, 182

Rykoff, E. S., Miller, J. M., Steeghs, D., \& Torres, M. A. P. 2007, ApJ, 666, 1129

Ryu, D., Chakrabarti, S. K., \& Molteni, D. 1997, ApJ, 474, 378

Shakura, N. I., \& Sunyaev, R. A. 1973, A\&A, 24, 337

Tomsick, J. A. 2010, ATel, 2384, 1

van der Klis, M. 2004, in Compact Stellar X-ray Sources, ed. W. H. G. Lewin, \& M. van der Klis (UK: Cambridge University Press)

van der Klis, M. 2005, AN, 326, 798

Yamaoka, K., Sugizaki, M., Nakahira, S., et al. 2010, ATel, 2380, 1

Yu, W. 2010, ATel, 2556, 1 